\title{
Lumen
}

Selected Proceedings from the Canadian Society for Eighteenth-Century Studies

\section{Sur les rives de l'Ohio : la cité utopique de Lezay-Marnésia}

\section{Roland Bonnel}

Volume 13, 1994

URI : https://id.erudit.org/iderudit/1012520ar

DOI : https://doi.org/10.7202/1012520ar

Aller au sommaire du numéro

Éditeur(s)

Canadian Society for Eighteenth-Century Studies / Société canadienne d'étude du dix-huitième siècle

ISSN

1209-3696 (imprimé)

1927-8284 (numérique)

Découvrir la revue

Citer cet article

Bonnel, R. (1994). Sur les rives de l'Ohio : la cité utopique de Lezay-Marnésia.

Lumen, 13, 43-59. https://doi.org/10.7202/1012520ar d'utilisation que vous pouvez consulter en ligne.

https://apropos.erudit.org/fr/usagers/politique-dutilisation/ 


\section{Sur les rives de l'Ohio: la cité utopique de Lezay-Marnésia}

On examinera en détail le plan et le fonctionnement de la communauté imaginée par le marquis de Lezay-Marnésia en 1790. Comme bien d'autres cités utopiques issues de l'imaginaire des Lumières, il s'agit $d^{\prime}$ 'une ville qui est le fruit des recherches en 'pathologie urbain $e^{\prime 1}$ menées par des architectes du XVIII ${ }^{\mathrm{e}}$ siècle comme Ledoux, mais aussi par bien des auteurs contemporains comme Rousseau et Restif de la Bretonne. C'est aussi une ville qui naît d'un processus de découverte ou de redécouverte géographique, institutionnelle et idéologique. La découverte est d'abord géographique car Gallipolis — c'est le nom de la future cité - sera bâtie sur des terres non défrichées au confluent de l'Ohio et $\mathrm{du}$ Scioto. La découverte est aussi institutionnelle car le fonctionnement de la communauté s'inspirera du modèle qu'offrent les dernières communautés taisibles existant encore en France et en Suisse, ainsi que de l'exemple des communautés moraves de Pennsylvanie. La découverte est également idéologique car Gallipolis offrira une nouvelle identité à une noblesse française dont les fonctions avaient perdu leur signification originelle. Finalement, il s'agit aussi d'une découverte en pathologie urbaine: celle d'un équilibre entre une ville purifiée et une société agricole communautaire dans un paysage qui est celui de la poésie descriptive à la Delille et des jardins paysagers à la Hubert Robert.

L'aventure commence au printemps 1790. Le terme 'aventure' n'est pas trop fort pour qualifier ce qui devait être l'expérimentation in vivo $d^{\prime}$ une utopie et la tentative de restauration d'un Age d'Or mythique. Le 26 mai 1790 deux navires quittent Le Havre et mettent la voile vers la Nouvelle Angleterre. A leur bord se trouvent 119 passagers dont 30 membres des classes privilégiées qui ont fait l'acquisition de terres sur les rives de l'Ohio. L'expédition est dirigée par le marquis de LezayMarnésia et le vicomte de Malartic. Les autres passagers, qui ne sont pas propriétaires, sont censés représenter les différentes professions dont on aura besoin pour constituer la colonie: cultivateurs, architectes, maçons, menuisiers, charpentiers, ecclésiastiques, etc. Ces deux navires ne sont pas les premiers à emporter les futurs colons de l'Ohio: deux navires ont 
quitté Le Havre en mars, deux au début de mai; un autre quittera Le Havre en août 1790 emportant le procureur du chancelier Duval d'Eprémesnil, qui fut l'un des principaux organisateurs de l'émigration vers les Etats-Unis. ${ }^{2}$ Ce sera le dernier navire car entre-temps sont arrivées les lettres des premiers colons dont la presse se hâte de se faire l'écho: 'les premiers fous partis de Paris pour les bords du Scioto sont dans la situation la plus déplorable. On en a trouvé réduits à demander l'aumône à New York. ${ }^{3}$

La colonie ne verra jamais le jour. Le petit groupe guidé par Marnésia et Malartic n'ira pas plus loin que Marietta car des dangers attendaient les nouveaux colons: des Indiens redoutables, une nature hostile et un hiver difficile. Ce sera la fin du rêve. La plupart des colons émigrent à La Nouvelle Orléans tandis que Marnésia et son fils s'installent à Pittsburgh. C'est là, en 1791, que Marnésia rédige ses Lettres écrites des rives de l'Ohio. Le rêve de Gallipolis reprend forme dans un cadre considéré comme idyllique. Il prend le nom d'Asylum pour rappeler les asiles anciens. Cette nouvelle tentative sera suivie d'un nouvel échec, cette fois pour des raisons financières. Ruiné par ses investissements dans le Scioto, Marnésia rentre en France en juin 1792. ${ }^{4}$

C'est un autre projet qui verra le jour, celui du vicomte de Noailles et d'Omer Talon qui poseront la première pierre d'une communauté modèle, une sorte de phalanstère avant la lettre, offrant aux émigrés le bonheur du Nouveau Monde sur 400.000 hectares situés sur les bords de la Susquehanna, en Pennsylvanie. ${ }^{5}$ Il s'agit de la ville d'Asylum (qu'il ne faut pas confondre avec l'Asylum de Lezay-Marnésia) dont l'un des fondateurs est Charles Boulogne qui avait été aussi l'agent général de la Compagnie du Scioto. Les premiers habitants s'installent dès 1795 et vont mener une vie mondaine dans un cadre rustique, semblable à celle que mène la marquise de La Tour du Pin dans sa ferme d'Albany où elle s'acquiert un grand succès en vendant des pains de beurre moulés à son chiffre. ${ }^{6}$ En 1801, la plupart des émigrés qui n'ont pas trouvé la nature idyllique à laquelle ils s'attendaient profitent de l'amnistie générale et rentrent en France.

Plus tard, en 1819, une autre colonie portant le nom de Champ d'Asile ou Aigleville verra le jour au Texas; elle sera fondée par des Français fuyant la Restauration. Pour eux aussi, il s'agira de trouver 'sur le globe un abri protecteur, un asile tutélaire et hospitalier. ' 'Ces Français, maîtres absolus de leurs pensées et de leurs opinions respirent librement aujourd'hui sous l'heureux ciel de l'indépendance,' lit-on sous une gravure représentant les travaux d'Aigleville commandés par le Général Lefebvre Desnouettes. ${ }^{7}$

L'aspect refuge n'est pas le seul caractère que ces colonies aient en commun. Ce sont aussi des fondations dont la valeur est sotériologique. 
A Champ d'Asile, on trouve les rues Austerlitz, Friedland et Wagram. A Asylum, on construit en plein centre de la communauté la Maison de la Reine. A Gallipolis, on prévoit que les rues, les places et les fontaines porteront les noms de célébrités françaises comme Pascal, Fénelon, Buffon, Rousseau, Corneille, Racine, Saint Vincent de Paul, Sully, Necker, Montesquieu. Ces noms ne sont pas choisis au hasard; leur valeur n'est pas seulement sotériologique comme dans les deux autres fondations; ils ont aussi une portée idéologique. Sully évoque l'essor de l'agriculture et Henri IV qui sous Louis XVI était présenté comme le modèle du roi de l'Age d'Or. Saint Vincent de Paul est mentionné pour ses actions charitables qui annonçent la bienfaisance; Fénelon, pour l'utopie aristocratique de Salente qui est l'un des modèles de Gallipolis; et Montesquieu, pour une tradition parlementaire qu'admirent les monarchiens de l'Assemblée nationale dont fait partie Lezay-Marnésia. Enfin, il y a la rue Necker. Cet homme cristallisait alors tous les espoirs monarchiens d'une régénération plus morale que politique comme le symbolise l'inscription portée sur la pyramide de la maison patriarcale et philosophique imaginée par Hupay de Fuvéa: 'A la patrie et à la religion rétablies par Necker. ${ }^{\prime 8}$ A Gallipolis, on trouvera même des rues Lally-Tollendal et Clermont-Tonnerre, deux hommes qui constituaient le cœur du groupe monarchien à l'Assemblée et partageaient avec LezayMarnésia la même conception de régénération du pays. A la différence d'Asylum et de Champ d'Asile, Gallipolis n'est donc pas purement conjoncturel; il s'agit d'un projet politique arrêté.

Le but est de résoudre la crise d'identité dont la noblesse fait l'expérience à la veille de la Révolution française. Entre les monarchistes intégristes et les nobles radicaux comme La Fayette, les gentilshommes éclairés offrent une solution modérée qui emprunte le véhicule de la bienfaisance. Leur programme de régénération n'est pas vraiment un discours politique; c' est un discours moral; c'est la bienfaisance en action. Il s'agit d'une thérapeutique paternaliste et sentimentale dont le registre lexical n'est pas celui de la justice mais celui de l'assistance. C'est cette pratique de la bienfaisance qui va donner à la noblesse une nouvelle légitimité. Les gentilshommes éclairés gèrent leurs domaines en suivant les conseils des agronomes et selon les enseignements de la poésie descriptive. Ils sont tous familiers avec Clarens. ${ }^{9}$ De cette pratique naît un néo-féodalisme, une idéologie de la protection qui n'est que la redécouverte du féodalisme à la lumière de la sensibilité. Dans une terminologie propre à la deuxième moitié du dix-huitième siècle, on retrouve les idées que Fénelon avait exposées dans ses Tables de Chaulnes. Il s'agissait de redonner à la noblesse son authenticité en en faisant une classe sociale utile. C'est en cela que Gallipolis est intéressante; au-delà d'un vernis pseudo-physiocratique, c'est une communauté qui met en 
œuvre les principes de cette idéologie néo-aristocratique et œuvre à la promotion d'un ordre terrien.

Le projet est issu du mouvement aristocratique du retour à la campagne qui s'amplifie à partir des années 1760 . Les seigneurs éclairés avaient quitté la cour pour retourner vivre à la campagne, dans leurs domaines, où ils avaient entrepris d'améliorer les conditions de vie des paysans et de perfectionner l'agriculture. Tel était le cas du marquis de Lezay-Marnésia qui suivait les exemples de La Rochefoucauld à Liancourt, de Tschoudi à Colombe ou de Buffon à Montbard. Mais cela ne suffisait pas. Il s'agissait aussi de prêter vie à l'idéal d'une société agricole communautaire. Ces nobles éclairés avaient lu Ovide, Virgile, Lucrèce et Horace; ils aspiraient au retour de l'Age d'Or qui apporterait avec lui le bonheur. Ils avaient aussi été élevés dans la mémoire et le respect des traditions ancestrales et dans le mythe de la protection médiévale que le seigneur devait à ses sujets. Ils rêvaient d'une société agreste qui rendrait tout le monde heureux car les relations humaines y seraient fondées dans la sensibilité et non sur des principes abstraits. Leur projet de régénération de la société, si libéral fût-il, restait basé sur une idéologie nobiliaire agraire et patriarcale. L'espoir de régénération et la nostalgie de l'Age d'Or étaient les deux aspects d'une même attitude réformiste. Cette pensée renforçait le rôle de l'aristocratie car elle remplaçait le pouvoir du roi par celui des seigneurs bienfaisants vivant à la campagne. Dans ce contexte, la fonction du citoyen se résume à l'obéissance, tandis que celle du noble s'en trouve accrue. Le noble est à la fois un modèle pour la nation, le protecteur du pauvre, l'ami du roi et l'intermédiaire entre le peuple et le monarque. Comme l'écrit Colardeau à propos des rapports de Duhamel de Denainvilliers avec ses paysans, 'il est leur juge, leur protecteur, leur ami et leur père. ${ }^{10}$ Aussi l'état de nature laisse-t-il la place à une nature qui perd sa pureté et son caractère thérapeutique si elle se trouve dépeuplée de nobles ou de propriétaires terriens comme à Gallipolis. A la place des bergers d'Arcadie, ce sont les nobles et les propriétaires qui peuplent cette campagne. Les leçons de la poésie descriptive ont porté; non seulement les nobles sont-ils retournés à la campagne pour se libérer de l'ennui et du vice des villes, mais ils ont aussi transformé le bonheur de la campagne qui n'aurait pas été complet sans leur bienfaisance.

Sur ce point, les gentilshommes éclairés ne faisaient qu'adhérer à un principe des Lumières, à savoir que le peuple a besoin de guides. $D^{\prime}$ un côté, la fonction de la noblesse est éducative; l'élite est avant tout un modèle pour la nation comme l'exprime Fresnais de Beaumont: 'nous verrons ces honnêtes laboureurs changer la face de nos campagnes (mais) il faut leur associer des gens aussi honnêtes, aussi actifs qu'eux, mais plus éclairés. ${ }^{11} \mathrm{D}^{\prime}$ un autre côté, la campagne est présentée comme 
un modèle de société et l'agriculture comme une école de vertu. On peut lire dans le Journal ceconomique que 'l'agriculture... est de toutes les occupations de l'homme la plus noble et la plus avantageuse... (qu'elle éloigne les cultivateurs) d'un grand nombre de vices, leur rend familière la pratique de beaucoup de vertus. ${ }^{12}$ Aussi paradoxal que cela soit, l'amalgame qui se produit à l'époque des Lumières entre l'éthique et l'esthétique de la campagne favorise chez les seigneurs éclairés l'invention d'un néo-féodalisme. Ce sont ces principes qui constituent la pierre d'angle de Gallipolis où l'agriculture servira de régulateur social.

Dès 1789 , un petit groupe d'aristocrates avait eu l'idée d'établir aux Etats-Unis une colonie sous le patronage de Bernardin de Saint-Pierre. Le choix des Etats-Unis n'était pas un hasard. Au cours des dernières années de l'Ancien Régime, la noblesse éclairée avait été nourrie de récits proposant une image idyllique du Nouveau Monde. Il ne s'agissait pas tant de l'Amérique de La Fayette, qui allait plutôt influencer la noblesse libérale, que de l'Amérique des agronomes ou de ceux qui se prenaient pour des agronomes. Des cavres comme les Voyages du marquis de Chastellux et les Lettres d'un cultivateur américain de Saint John Crèvecœur mettaient en valeur - et exagéraient — les vastes possibilités que le nord-est des Etats-Unis pouvait offrir aux personnes qui rêvaient d'expériences agricoles modernes. La nouvelle nation semblait offrir les promesses d'une société régénérée. En dépit des épreuves rencontrées, Marnésia s'extasiera toujours devant le spectacle offert par les plantations de Pennsylvanie. La description idyllique qu'il en fait dans ses Lettres écrites des rives de l'Ohio est visiblement inspirée par Chastellux et Crèvecœur. Les cabanes sont devenues de grandes demeures et les bibliothèques privées contiennent les œuvres de Thomson, de SaintLambert, de Delille et de Fontanes. Le paysage qu'il décrit est plus proche du jardin d'Eden que de la réalité pennsylvanienne; ${ }^{13}$ mais peu importe, car ce qu'il veut offrir, c'est un paysage moral. Esthétique et éthique restent inséparables. Il ne cache pas d'ailleurs les souvenirs littéraires qui se mêlent dans son esprit lorsqu'il découvre la campagne américaine qui lui semble si propice à la restauration de l'Age d'Or. S'il se réclame de La Nouvelle Héloïse, il invoque aussi La Reine de Golconde du chevalier de Boufflers, la Salente de Fénelon, les Troglodytes de Montesquieu et les Etudes de la Nature de Bernardin de Saint-Pierre. Ainsi rêve-t-il d'aider les personnes en quête du bonheur en créant une colonie 'dans un désert du nouveau monde pour leur faire couler des jours paisibles et purs dans les douceurs de la vie patriarcale, dans les charmes de la vie fraternelle... (et pour) se livrer au bonheur d'adorer Dieu, de pratiquer les vertus que la religion enseigne, commande et récompense, même en cette vie. ${ }^{14}$ Cette tendresse - le mot n'est pas trop fort - présidera à l'organisation de la vie à Gallipolis qui sera ponctuée de fêtes et de banquets rappelant 
l'étrange table patriarcale décrite par Hupay de Fuvéa ${ }^{15}$ et annonçant les banquets de la poésie de Marchangy. ${ }^{16}$

Quel simple et magnifique banquet! Sous des rameaux chargés de touffes de fleurs superbes, au milieu des plantes aromatiques et suaves, sur des gazons qui rassemblent les plus tendres et les plus vives couleurs toujours rafraîchies par les rosées abondantes et par les eaux limpides des petits ruisseaux, seront servies les viandes succulentes des troupeaux nourris dans les gras pâturages de la nature.... Le sourire sera sur toutes les lèvres, le plaisir brillera dans tous les yeux; l'émotion et la tendresse seront dans tous les cœurs. ${ }^{17}$

Dans ce cadre rural, les Français d'Amérique pratiqueront une chaîne d'union et prononceront 'le serment de s'aimer d'une amitié sincère, active, inaltérable et sans cesse renaissante. ${ }^{18}$

A la différence d'Asylum et de Champ d'Asile qui sont issus directement de l'émigration, le projet de Marnésia naît de la spéculation philosophique. La Révolution et la disponibilité de terres sur les bords de l'Ohio ne sont que des accidents qui hâtent le processus de réalisation. En 1789, une compagnie immobilière, la Compagnie du Scioto, ouvre des bureaux à Paris dans le but de vendre à des investisseurs et à des colons potentiels des terrains situés à l'ouest des Montagnes Bleues. Un petit groupe de propriétaires terriens connu sous le nom de la Société des Vingt-Quatre fait l'acquisition de 24.000 acres pour créer une communauté idéale. Parmi les acquéreurs on trouve des personnalités en vue dans les milieux politiques: Duval d'Eprémesnil, Lezay-Marnésia, Malartic, Lally-Tollendal, Mounier, Malouet, Vichy. Sauf Duval d'Eprémesnil, tous sont proches du groupe des monarchiens. La plupart sont également des magistrats, ce qui fait dire à un pamphlétaire de 1790 que le Scioto est la coqueluche des 'sociétés robinocrates. ${ }^{19}$ Ils ont tous un autre point commun: ce sont des philanthropes qui rêvent de régénération, de bonheur et de restauration d'un Age d'Or qu'ils placent dans l'ancienne constitution de la France sans toutefois trop en préciser la nature. De tous ces propriétaires, seuls Marnésia et Malartic traverseront l'Atlantique. Au cours du premier semestre 1790, l'entreprise du Scioto a droit aux honneurs de la presse, autant à cause de l'ampleur du projet que de la personnalité de d'Eprémesnil qui catalyse alors les critiques. La Chronique de Paris s'alarme du nombre croissant de personnes de toutes les professions qui s'inscrivent pour accompagner quelques grands seigneurs au Scioto et invoque l'argument du patriotisme pour faire cesser cette émigration..$^{20}$ Dans un article du 15 mars, Cloots stigmatise l'imagination des aristocrates qu'ont exaltée les romans de Saint John Crèvecœur. 
Nos dames délicates qui se condamnent à cet exil avec le délire de la fièvre chaude auront le temps de se repentir. Aucune métamorphose charmante ne se dérobe à leurs idées chimériques. Les arbres se transforment en palais; les hordes sauvages en bergers tendres; la misère, la douleur, l'ennui, la mort prématurée en une perspective riante de longues jouissances physiques et morales.... Il sera trop tard d'écouter la raison lorsque leur chevelure servira de trophée à des barbares qui enlèvent subitement le péricrâne aux paisibles laboureurs. Rien de plus beau que les projets enfantés au coin de son feu. ${ }^{21}$

Au delà de la satire politique, l'argumentation repose sur la réalité. Dans quatre longs articles du Spectateur National on offre un antidote aux tableaux flatteurs et riants de Crèvecœur. On y brosse non seulement un tableau réaliste de la situation en Amérique, mais aussi on y dénonce les activités des représentants de la Compagnie du Scioto qu'on traite de charlatans. ${ }^{22}$ Cette accusation est reprise par l'ensemble de la presse.

Paris est inondé de spéculateurs charlatans qui vont prônant une Compagnie du Scioto... et qui trouvent des dupes à Paris. Ces Messieurs vendent ici tant d'âcres de terre américaine, argent comptant, comme Saint Bernard vendait aux imbéciles de son siècle tant $\mathrm{d}$ 'arpents de terrain en Paradis. ${ }^{23}$

Ces critiques avaient un double but. Il ne s'agissait pas seulement de mettre à jour les actes malhonnêtes des agents de l'entreprise immobilière américaine, mais aussi de détruire l'image d'une Amérique idyllique qui existait dans l'imaginaire collectif. Ce fut suffisant pour décourager ceux qui cherchaient un moyen d'échapper aux événements révolutionnaires tout en réalisant une spéculation financière. Pour Marnésia, toutefois, la spéculation était philosophique comme le prouvent sa correspondance privée et les inscriptions qui seront portées sur la colonne commémorative qu'on dressera au centre de Gallipolis. D’un côté de la colonne: les noms des fondateurs; de l'autre côté cette inscription:

Ils sont venus avec des cœurs bons et sensibles

Dans l'intention

De pratiquer les douces vertus,

Et pour y jouir

De la paix de la liberté véritable

Et des charmes de la fraternité,

Y vivre dans la simplicité primitive,

Et y recueillir,

En se livrant à un travail actif et modéré,

Tous les dons de l'agriculture. ${ }^{24}$ 
Cette colonne commémorative se dresse au centre d'un espace dont l'organisation reflète l'idéologie qui préside à la fondation de la communauté. La description détaillée de ce qu'aurait été Gallipolis présente un plan d'organisation de l'espace qui traduit physiquement le caractère à la fois patriarcal et éclairé du projet de société.

Lezay-Marnésia procède à la façon d'un utopiste. Gallipolis est une société qui se suffit à elle-même et qui doit être protégée de l'influence extérieure, du moins au départ, pour assurer sa réussite. Avant de partir, il écrit à sa femme qu'il construira des murs autour de la ville et qu'il fera de nouvelles lois, comme Idoménée à Salente. ${ }^{25}$ On est même dans le domaine de l'idylle comme le prouvent les descriptions des paysages et des relations entre les habitants des lieux: les paysages sont riants, les relations sont harmonieuses. Le projet est l'expression politique d'une sensibilité qui s'émeut à lire les descriptions de Clarens tout autant que celles de l'univers de Paul et Virginie.

La société qui émerge de ces principes est à la rencontre des idées des Lumières et de la pensée populiste de la noblesse. Dans cette société règnera l'égalité; pas l'égalité absolue mais seulement l'égalité naturelle décrite par Jaucourt dans l'Encyclopédie. Il s'agit d'une égalité qui ne met pas en danger l'existence d'une hiérarchie fondée sur le mérite, l'utilité sociale et le rang. Dans cette société, le contrat affectif entre les citoyens et les seigneurs-propriétaires sera plus fort que l'égalité. A une notion abstraite et universelle on préfère une relation concrète et sensible. L'égalité sera celle qui existe au sein du modèle familial.

Gallipolis partage le modèle néo-aristocratique et le modèle familial avec bien d'autres utopies. Par exemple, la Salente de Fénelon pour le modèle aristocratique, la Mezzoranie de Berington ${ }^{26}$ pour le modèle familial. Gallipolis tient à la fois de l'une et de l'autre. Ces sociétés utopiques ont en outre une économie surtout agricole où la présence de toute forme de luxe a été bannie. La vie y est frugale, la production limitée au nécessaire, l'organisation de la vie et les vertus soumises à l'utilité sociale. Il n'est pas jusqu'à l'espace qui ne soit comparable. Ce sont d'immenses jardins au milieu desquels on a construit des citésjardins. La propriété est privée; mais à Gallipolis, comme en Mezzoranie, il existe une forme de propriété communale - ou plutôt familiale - qui en transforme l'apparence et en fait un bien public.

Le gouvernement lui-même est de nature patriarcale. En Mezzoranie, il est strictement familial, le pouvoir appartenant à une tribu. A Gallipolis, l'aspect patriarcal s'accommode des idées physiocratiques diffuses dans l'opinion éclairée; le produit final proposé par Lezay-Marnésia est celui d'une organisation à la fois aristocratique et communautaire. La cellule de base de la colonie est la famille au sens antique du terme. Le gouvernement de la colonie appartient à l'union des propriétaires asso- 
ciés, ce qui lui donne par bien des aspects l'apparence d'une multi-propriété. Les familles sont au cœur de la structure sociale de la colonie. Leur nombre est limité à cinquante-deux, mais peut être porté à cent. Si elles possèdent des propriétés agricoles, elles ont aussi une spécialisation en fonction des besoins de la communauté: 8 familles s'occuperont de chapellerie, 20 de la production de toiles et 24 de la production de draps. Ces familles choisissent parmi elles douze administrateurs renouvelables par quart tous les ans. Toutefois, seuls les propriétaires, c'est-à-dire les chefs de famille, peuvent être élus à ces fonctions. Si l'égalité entre les propriétaires et les salariés est mentionnée, elle ne s'applique pas au niveau de l'administration. D'une part parce que les salariés sont employés sous contrat; et se trouvent donc être des 'citoyens sous contrat' dont les droits sont strictement définis. Les propriétaires devront à leurs salariés 'des soins, de la protection, le prix abondant de leur travail, de la douceur, de l'indulgence et de la bonté. Rien au delà. ${ }^{, 27} \mathrm{D}^{\prime}$ autre part parce qu'il n'est pas possible de mettre côte à côte les propriétaires et leurs salariés, fermiers et artisans, en raison de leurs intérêts différents et de leurs différences de ressources et d'éducation. Cette hiérarchie s'inscrira, on le verra ci-dessous, dans l'organisation de l'espace: vivre dans la ville ne sera autorisé qu'aux propriétaires.

L'utopie marnésienne s'inscrit sans aucun doute dans le débat sur le luxe. Elle peut aussi s'interpréter à la lumière des discussions de l'époque sur le bien fondé de la participation de la noblesse aux activités commerçantes. Elle est aussi issue d'une réflexion sur la régénération de la nation. Ces différentes lectures se recoupent. On insistera plus particulièrement sur l'aspect de communauté agricole car il est issu d'une redécouverte faite par le dix-huitième siècle: l'existence de sociétés rurales harmonieuses. ${ }^{28} \mathrm{Ce}$ sont d'une part les communautés taisibles et d'autre part les fondations créées par des sectes religieuses comme les frères Moraves.

Les communautés taisibles de la région de Thiers sont les plus connues car elles font l'objet d'un article spécial dans le Cours complet d'agriculture de Rozier, mais aussi parce qu'elles ont le privilège d'être mentionnées par Voltaire et Rétif de la Bretonne. Toutefois, c'est au Journal œeconomique qu'on doit la découverte, dès 1755 , de ces communautés qui annoncent sous bien des aspects les phalanstères de Fourier ou les communautés de Cabet. ${ }^{29}$ Très rapidement, une sorte d'assimilation se produit entre ces communautés et les établissements des Moraves, comme le montrent l'article 'Moraves' de l'Encyclopédie et l'article 'Economie Domestique' que Voltaire fait paraître dans son Dictionnaire philosophique. Ce qu'on retient de ces sociétés qui font figure d'utopies en action, c'est plus l'aspect moral et communautaire que l'aspect agricole, ou si on souligne le travail de l'agriculture, $c^{\prime}$ est pour mieux 
montrer comment il engendre la vertu. 'Les statuts du Bourg d'Oudun,' que Rétif de la Bretonne imagine à la fin du Paysan perverti, révèlent bien que ce qu'on recherche est une leçon morale et sociale. C'est ce que fait aussi Hirzel dans son Socrate rustique où il décrit la société de Jacques Gouyer. ${ }^{30} \mathrm{~S}^{\prime} \mathrm{il}$ parle des progrès en matière de fumier, de prairies artificielles, de navets et de pommes de terre, c'est pour mieux montrer que les progrès de l'agriculture passent par une réforme morale. Le marquis de Pezay fait la même constatation lorsqu'il rend visite aux communautés anabaptistes de la région de Montbéliard et aux Bousbots de Franche-Comté. ${ }^{31} \mathrm{Il}$ affirme que leur prospérité provient de leurs mœurs qu'il résume en quatre points: l'importance accordée au principe d'utilité, la pratique des vertus qui rend pur, l'absence de toute forme de luxe, la grande propreté de l'habitat. Pezay en tire une loi générale: 'on doit la population à l'abondance, l'abondance à la culture perfectionnée, la perfection de la culture aux bonnes meurs et aux bonnes lois. ${ }^{32}$ L'éthique rejoint l'esthétique, et les Bousbots rappellent à Pezay les bergers du Lignon que la réalité fait souvent oublier. Propreté et bonnes mœurs sont aussi présentes dans l'utopie marnésienne. Lezay-Marnésia connaissait les anabaptistes de Montbeliard et les Bousbots. Toutefois, ce qu'il doit à ces sociétés rurales, ce n'est pas seulement une leçon morale - elle baigne tous les ouvrages consacrés à l'agriculture - c'est aussi une organisation de la vie sociale. Les agronomes ne se font pas faute de citer en exemple ces communautés qui représentent à leurs yeux la matérialisation de leur idéal d'une société patriarcale.

Ces communautés agricoles qui datent du quatorzième siècle sont en voie de disparition au dix-huitième siècle, surtout en France où, en dépit de ce que dit le marquis de Pezay, leur agriculture reste rudimentaire. Cependant leur organisation reste inchangée. Les terres sont la propriété de la communauté qui est constituée par une seule famille. Un tel système d'indivision ne pouvait fonctionner que sur la base de l'utilité, et ainsi favoriser le bien général. A la tête de la communauté se trouve un chef de famille; il est élu en fonction de son aptitude à s'occuper des affaires de la communauté. Par ailleurs, les différentes cellules familiales - dont le nombre est limité - ont chacune, outre l'agriculture, un métier qui leur est spécifique car il s'agit de sociétés autarciques. Les caractéristiques peuvent varier d'une communauté à l'autre; ce qui demeure c'est la relation sociale qui est de nature familiale. Il s'agit de relations affectives et tendres qui engendrent une union intime entre les membres. La sensibilité se trouvait ainsi érigée en une idéologie politique qui servait la représentation que la noblesse éclairée se faisait du contrat féodal.

Les communautés des frères moraves allaient renforcer ce modèle, et on ferait silence sur leur but religieux au profit d'un discours sur l'harmonie et le bonheur. ${ }^{33}$ En 1765 , l'article de l'Encyclopédie avait suscité 
l'intérêt pour ces sociétés rurales harmonieuses. La redécouverte de l'Amérique ne fit que relancer cet intérêt. Bethléem est mentionné par La Fayette dans sa correspondance. Chastellux en fait une longue description. Il la décrit comme une société monacale où chacun apprend un métier et où les maisons sont simples, jolies et entourées de jardins cultivés avec soin. Des Moraves, on ne va retenir que l'aspect idyllique. Lezay-Marnésia voit Bethléem comme 'le séjour de la paix, de la sagesse et de la tranquille félicité. ${ }^{34} \mathrm{Il}$ la décrit comme une société d'abondance, une société riche et confortable sans qu'il y ait la moindre trace de luxe, une société où les gens sont honnêtes, hospitaliers et ne cherchent qu'à se rendre utiles. On voit ainsi se profiler la nostalgie de l'Age d'Or dans la description d'une communauté morave vidée de son caractère religieux. En outre, le Bethléem que visitent Chastellux et Lezay-Marnésia n'est déjà plus le Bethléem des années 1740 ni celui de l'Encyclopédie. Le système des chœurs a laissé place à plus d'intimité familiale: les enfants sont élevés par leurs parents qui de plus en plus optent pour la maison individuelle plutôt que pour l'habitat communautaire. Enfin la propriété foncière est privatisée en 1766 et tous les établissements commerciaux le sont en 1769. Dans sa Gallipolis, Lezay-Marnésia reproduira le système mixte de l'économie morave de la fin des années 1760: les propriétaires possèderont en propre leurs terres à la campagne, mais les manufactures et les maisons des banlieues et de la ville seront la propriété de la société des propriétaires. Ce que les visiteurs retiennent de cette communauté, c'est l'intégration totale de l'individu à la collectivité et une harmonie qui est réalisée plus par un rapport d'affectivité que par des idées, la religion équilibrant la raison. Les visiteurs sont également frappés par la communauté de travail où une tâche bien précise est assignée à chaque Morave en fonction de ses aptitudes. La communauté est fondée sur une éthique du travail qui s'exprime dans des fêtes de l'amitié et dans des repas pris en commun pour célébrer tout ce qui se rattache au travail. Les banquets de Gallipolis doivent sans doute beaucoup à ces agapes moraves. Quant à la dévotion religieuse, les visiteurs n'en retiennent que l'image idyllique d'une communauté qui chante en travaillant et d'un processus de socialisation où l'intérêt commun a plus d'attrait que l'intérêt particulier. ${ }^{35}$

Toutefois, l'organisation de l'espace ne suit pas la structure carrée que les Moraves avaient donnée à leur ville. Saint-Pierre, la capitale de Gallipolis, sera bâtie en demi-cercle. Il s'agit d'une communauté fermée, composée de cinq zones. A l'extérieur, enveloppant la ville, s'étendra la campagne, divisée en domaines privés de 1.500 acres chacun. Elle offre un paysage à la Delille, rappelle les jardins paysagers d'Hubert Robert et les fermes ornées de Watelet ou de Oudry. 
De la campagne on passera à une zone semi-urbanisée où se trouveront l'hôpital des Sœurs de Saint Vincent de Paul et les manufactures. Comme le recommandaient les architectes du dix-huitième siècle, ces bâtiments se trouveront à la périphérie de la ville. Il est important aux yeux de Lezay-Marnésia d'éviter la tentation du luxe que peuvent engendrer les centres de production qui, pour la même raison, ne produiront qu'à partir des matières produites localement: draps, toiles, chapeaux, cuirs. Par ailleurs, la ville sera ainsi protégée des artisans et des ouvriers qui sont souvent querelleurs et ivres. Saint Pierre sera une ville régénérée où il n'y aura que de beaux objets et où ne règnera que le calme le plus doux.

Entre la ville proprement dite et la zone industrielle se trouveront les faubourgs. Il s'agira d'une ceinture de maisons modestes et propres qui appartiendront à la société des propriétaires. Ces maisons seront le moyen de récompenser les familles d'artisans et d'ouvriers qui se montreront laborieuses et sages. Il est intéressant de remarquer que LezayMarnésia n'envisage ni contrôle ni surveillance des ouvriers. Sur ce point, il prend nettement ses distances par rapport aux architectes des Lumières. La forme en demi-cercle qui favorise la surveillance aux Salines d'Arc-et-Senans a une autre fonction dans le projet marnésien. La surveillance s'exerce plutôt dans le style affectif de Clarens. Il y aura à Gallipolis une catégorie de magistrats, appelés les 'approbateurs,' dont la seule fonction sera d'évaluer les actions des ouvriers en termes d'utilité, de bonne conduite et de vertu. Chaque année, le jour de la fête de Saint Jean l'Apôtre, seront publiés les noms des travailleurs qui l'auront mérités. Comme récompense, ils recevront l'usage, pour un an, d'une de ces petites maisons des faubourgs. Ils se trouveront ainsi plus près du Paradis que semble représenter la ville dans cette société régénérée.

A l'intérieur des murs d'enceinte, autour d'une place en demi-cercle, se dresseront des 'maisons sans faste, sans décoration, mais riantes, agréables et même élégantes... Toutes devront avoir des jardins (car) autant il est sage de ne pas porter dans les champs le tableau des villes, autant il est raisonnable de retrouver dans les villes les images des champs. ${ }^{36}$ Ces maisons serviront aux propriétaires quand ils se rendront en ville pour affaires. Ils y passeront aussi l'hiver. Ainsi se trouve transporté sur les rives de l'Ohio le mode de vie du gentilhomme passant l'hiver à Paris à s'occuper des arts et l'été à la campagne à soigner ses domaines. La population de la ville sera donc toujours petite, sauf l'hiver: l'évêque et ses douze prêtres, cinq magistrats, les douze chefs de famille chargés de l'administration des manufactures et les professeurs des collèges et leurs élèves. 
En plein cœur de la cité il y aura une place semi-circulaire autour de laquelle seront construits les bâtiments et les monuments les plus importants de la colonie. Tout d'abord la colonne commémorative de la fondation dont on a vu la fonction idéologique: rappeler les principes fondateurs, comme la colonne trajane des jardins du marquis de Laborde-Méréville qui avait été dressée pour rappeler les exploits accomplis outre-mer par la famille. En face de la colonne: l'église catholique, appelée Temple dans le texte de Marnésia, flanquée des bâtiments paroissiaux et du palais épiscopal où le clergé vivra en communauté. L'église sera le seul bâtiment luxueux de la colonie. Sur son fronton seront gravés les mots 'Doctrine et Vérité'; sur ses colonnes, les Tables de la Loi; pour la décoration intérieure, il sera fait appel à tous les arts. De chaque côté de l'église se trouveront d'une part le Palais de Justice, d'autre part le Centre commnautaire. C'est dans ce centre communautaire qu'auront lieu les spectacles, les marchés, les assemblées générales et les banquets où tous se retrouveront, $\mathrm{y}$ compris les ouvriers agricoles et ceux des manufactures qui ainsi 'feront mieux que de se donner des égaux, ils se donneront de tendres et de constants amis. ${ }^{37} \mathrm{Le}$ Centre communautaire sera donc plus qu'un marché; il sera un lieu de propagande, la matérialisation de la sensibilité conçue comme outil politique. Enfin, aux extrémités de la place, le collège des garçons et celui des filles où ils apprendront, outre les disciplines traditionnelles, la médecine, les mathématiques, la botanique, la chimie, la musique et le dessin.

Cet espace sacré ne sera donc pas seulement le cœur géographique de la communauté, mais aussi son centre idéologique. Ce sera le lieu de l'initiation. Les jeunes vivront dans un univers limité par la bienfaisance rappelée par la colonne, la religion incarnée par l'église, la justice symbolisée par le palais de justice et l'harmonie matérialisée par le centre communautaire. La fonction d'un espace semi-circulaire prend alors toute sa signification. Il ne s'agit pas de surveiller mais d'inculquer par l'expérience de l'espace un sentiment d'harmonie et d'unité. L'espace devient idéologique. Les modèles d'espace semi-circulaires ne manquaient pas, à commencer par celui des Salines d'Arc-et-Senans. Quelqu'en soit le symbolisme, la forme semi-circulaire appliquée à une ville favorise le centralisme. S'il y a centralisme à Gallipolis, ce n'est pas au niveau d'un gouvernement exécutif dont on ne connaît même pas l'emplacement, c'est plutôt au niveau idéologique: l'harmonie. La forme semi-circulaire de Gallipolis, tout comme la table en arc de cercle du repas patriarcal d'Hupay de Fuvéa, renforce le sentiment d'appartenance à la communauté.

Gallipolis fait ainsi figure d'une cité morale, sans corruption, sans luxe, sans surpopulation. Son esthétique et l'organisation de son espace 


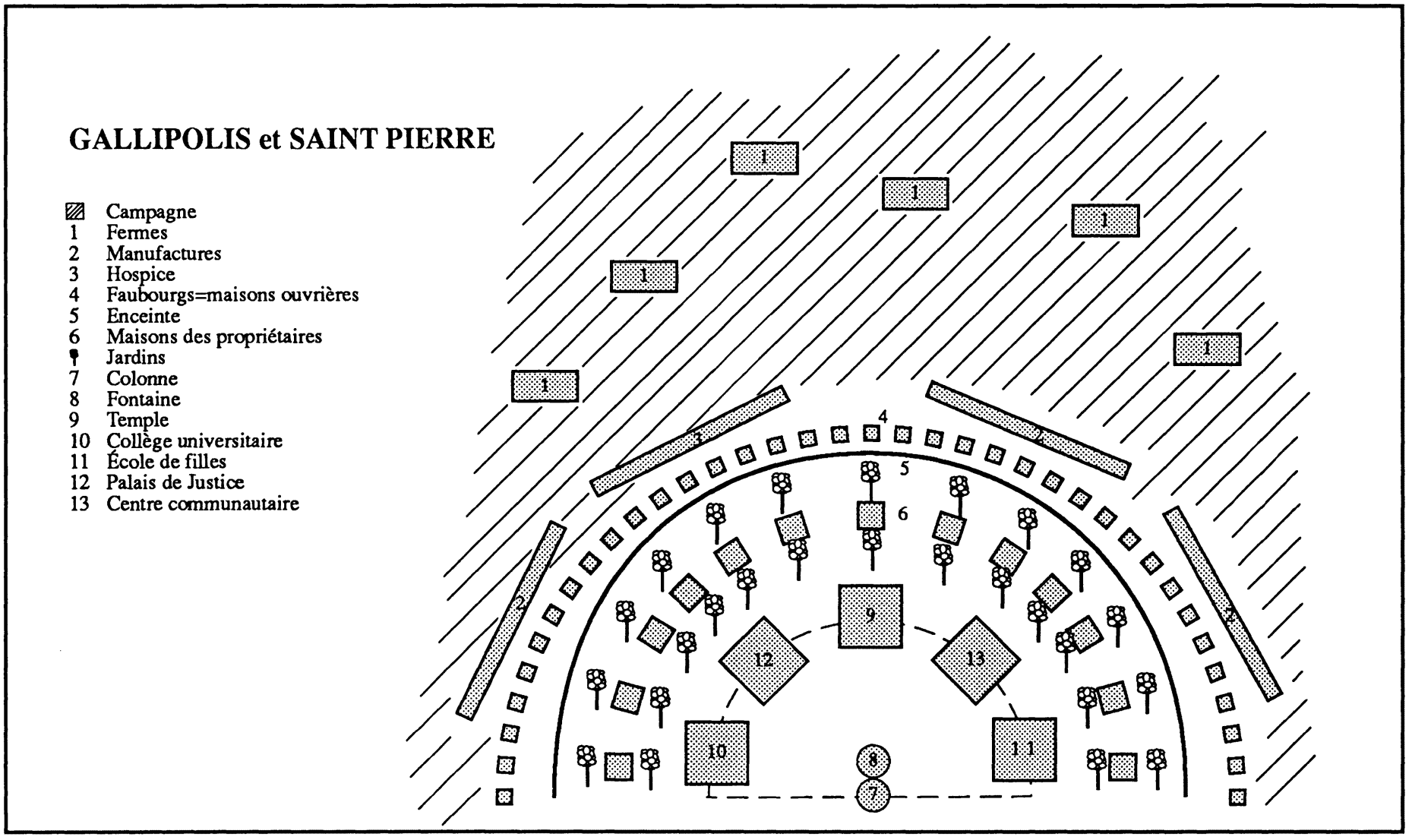


véhiculent une signification éthique. Les bâtiments du sanctum sanctorum ne sont pas seulement fonctionnels; ils ont aussi une portée allégorique. La ville régénérée est l'inverse de la ville corrompue, de ces 'grandes latrines' dont parlait le marquis de Pezay. Elle retrouve ses fonctions premières de lieu d'instruction et $\mathrm{d}^{\prime}$ initiation ${ }^{38}$ La campagne devient ainsi la garantie de la pureté de la ville, et la ville ce qui sauve la campagne. C'est pourquoi, à l'image des communautés moraves, Gallipolis sera, tout comme Chaux, une cité-jardin.

$\mathrm{Au}$ début de ses Lettres, le marquis de Lezay-Marnésia appelait à l'action. 'Ne ferons-nous donc jamais que des livres?... Nous borneronsnous sans cesse par de grandes et belles idées à exciter une admiration stérile, à faire couler quelques heures délicieuses dont il ne reste que de faibles traces dès que le livre est fermé $?^{\prime 39}$ Cette communauté de Gallipolis qui a failli voir le jour représente la matérialisation de la bienfaisance. Paradoxalement, elle est aussi la traduction architecturale d'un ordre où le pouvoir de la noblesse terrienne repose sur un contrat affectif par lequel les sujets abandonnent jusqu'à leurs pouvoirs de citoyens en contre-partie de la bienfaisance des nobles. Ainsi la redécouverte des sociétés rurales harmonieuses avait-elle permis d'inscrire dans l'organisation d'un espace une structure sociale patriarcale et néo-féodale.

\section{ROLAND BONNEL \\ Dalhousie University}

\section{Notes}

1 L'expression est utilisée par Mona Ozouf dans son étude L'Ecole de la France. Essais sur la Révolution, l'utopie et l'enseignement (Paris, 1984), p. 299.

2 Pour l'émigration vers les Etats-Unis, voir Henri Carré, 'Les émigrés français en Amérique, 1789-1793,' La Revue de Paris, 15 mai 1898, pp. 311-340; Bernard Faÿ, The Revolutionary Spirit in France and America. A study of moral and intellectual relations between France and the United States at the end of the eighteenth century (London, 1928); J.G. Rosengarten, French Colonists and Exiles in the United States (Philadelphia and London, 1907). La Gallipolis dont rêve Lezay-Marnésia et que nous étudions ici est restée un rêve; la réalité, la cité de Gallipolis, composée des cabanes construites par les hommes du major Burnham pendant l'été 1790 à l'intention des colons français, dont 500 finiront par arriver à l'automne, n'a rien à voir avec l'utopie marnésienne. Voir Roland Bonnel, Ethique et esthétique du retour à la campagne au XVIII siècle. L'ouvre littéraire et utopique de Lezay-Marnésia, 1735-1800 (New York and Frankfurt: Peter Lang, à paraitre en 1995).

3 Journal Général de la Cour et de la Ville, 7 mai 1790, p. 292.

4 Toutefois il n'abandonne pas son idée. Une lettre d'août 1792 nous apprend qu'il envisage de retourner sous peu aux Etats-Unis et qu'il attend une décision du Congrès américain sur son établissement. Dans cette lettre il est question de la 
création d'une colonie basée sur les mêmes principes que Gallipolis. Marnésia prévoit désormais de construire une ville au confluent de l'Illinois et du Mississipi, et de la protéger des sauvages et de la contrebande par un fort militaire. Cette lettre est signée par M. Audrain, un français établi au Fort Pitt qui s'était pris d'amitié pour Lezay-Marnésia et lui avait offert l'hospitalité lorsque celui-ci se trouvait dans le besoin (selon les renseignements fournis par Marnésia lui-même dans ses Lettres écrites des rives de l'Ohio [Paris \& Fort Pitt, an ix], p. 143). Elle est datée du 18 août 1792 et adressée à Lezay-Marnésia à Londres, où le marquis s'est arrêté sur le chemin du retour. Archives Lezay-Marnésia, MSS. 7326, Bibliothèque de l'Arsenal, Paris.

5 Voir Ghislain de Diesbach, Histoire de l'émigration (Paris, 1984), pp. 507-512 et La Rochefoucault-Liancourt, Voyage dans les Etats-Unis d'Amérique fait en 1795, 1796 et 1797 (Paris, an vii).

6 Marquise de La Tour du Pin, Mémoires (Paris, 1989), p. 206.

7 Ce même désir de trouver un asile se retrouve dans la correspondance que Lezay-Marnésia adresse à sa femme avant son départ. Cette correspondance se trouve encore dans des archives privées. Toutefois, de larges extraits en ont été publiés dans l'étude d'Elisabeth Bourget-Besnier, Une famille française sous la Révolution et l'Empire, la famille de Lezay-Marnésia (Paris, 1985).

8 J.A.V. Hupay de Fuvéa, Généralif, maison patriarcale et champêtre (Aix-en-Provence, 1790), p. 12.

9 Pour l'aspect aristocratique de La Nouvelle Héloïse, voir: Jean Biou, 'Le rousseauisme, idéologie de substitution,' Roman et Lumières au XVIII siècle (Paris, 1970), pp. 115-128; Renato Galliani, Rousseau, le luxe et l'idéologie nobiliaire (Oxford, 1989); Norbert Sclippa, 'La Nouvelle Hélö̈se et l'aristocratie,' Studies on Voltaire and the Eighteenth Century, 284, 1991, pp. 1-72.

10 Colardeau, Epître à Monsieur Duhamel de Denainvilliers (1774), in CEuvres de Colardeau (Paris, 1779), ii, p. 269.

11 Fresnais de Beaumont, La Noblesse cultivatrice (Paris, 1778), pp. 4-5.

12 Journal œconomique, janvier 1751, pp. 6-7.

13 Cf. John Francis McDermott, 'John B.C. Lucas in Pennsylvania,' Western Pennsylvania Historical Magazine, xxi, September 1938, pp. 210-211; and Hugh G. Cleland, 'John B.C. Lucas, Physiocrate,' Western Pennsylvania Historical Magazine, xxxvi, March 1953, pp. 3, 6.

14 Lettres écrites des rives de l'Ohio (Paris \& Fort Pitt, an ix), pp. 14-15.

15 Hupay de Fuvéa, Généralif, pp. 25-27.

16 Louis-Antoine-François de Marchangy, Le Bonheur, poëme en quatre chants (Paris, 1804), p. 52.

17 Lettres écrites des rives de l'Ohio, pp. 49, 55.

18 Lettres écrites des rives de l'Ohio, p. 57.

19 Le Parlement de Paris établi au Scioto (Paris, 1790), p. 13.

20 Chronique de Paris, no. 11, 11 janvier 1790, p. 42; no. 32, $1^{\mathrm{er}}$ février 1790, p. 127.

21 Chronique de Paris, no. 74, 15 mars 1790, p. 294.

22 Le Spectateur National, no. xxvii, 27 janvier 1790, pp. 105-106; no. xxviii, 28 janvier 1790, p. 109; no. xxix, 29 janvier 1790, pp. 113-114; no. xxx, 30 janvier 1790, p. 117. 
23 Journal Général de la Cour et de la Ville, no. lxv, 6 mars 1790, p. 516.

24 Lettres écrites des rives de l'Ohio, pp. 70-71. Ce projet d'inscription avait été adopté par la Société des Vingt-Quatre avant même le départ des premiers colons.

25 Lettre du 9 novembre 1789, citée par Bourget-Besnier, Une famille sous la Révolution, p. 26.

26 Le livre de Simon Berington, Mémoires de Gaudence de Lucques, avait été traduit en français en 1746 et à nouveau en 1753.

27 Lettres écrites des rives de l'Ohio, p. 74.

28 Sur ce point, voir André Bourde, Agronomie et Agronomes en France (Paris, 1967), pp. 1062-1076; Charles Rihs, Les Philosophes utopistes (Paris, 1970), pp. 320-333; Bronislaw Braczko, Lumières de l'utopie (Paris, 1978), pp. 58-59.

29 Journal œconomique, septembre 1755, p. 97; mars 1756, p. 81; juillet 1756, p. 85.

30 Hirzel, Le Socrate rustique (Zürich, 1762), p. 80.

31 Marquis de Pezay, Les soirées helvétiennes, alsaciennes et franc-comtoises (Paris, 1771), pp. 40-48 et pp. $125-42$.

32 Ibii.., p. 40.

33 L'Ecole civile et militaire de Goyon de la Plombanie (La France agricole et marchande, Avignon, 1762, ii, pp. 409-439) révèle déjà l'influence des communautés taisibles et des communautés moraves sur l'imaginaire des Lumières. Goyon était journaliste au Journal æconomique et avait donc connaissance des articles publiés sur ces communautés. Son école semble être une adaptation du système des chœurs séparés pratiqué dans les communautés moraves de Herrnhut et de Bethléem. L'école est conçue pour promouvoir l'agriculture et le commerce pour les roturiers et le métier des armes pour les nobles. Dans cette école, la séparation des individus ne se fait pas uniquement selon la catégorie sociale, mais aussi en fonction du sexe, de l'âge, du statut marital et du métier. Chaque groupe est isolé. L'aspect le plus étrange de ce projet en est la traduction architecturale. Il s'agit d'une forteresse à la Vauban pouvant contenir 250.000 âmes. Elle est constituée d'enceintes de huits étages (les bâtiments des chœurs moraves de Bethléem avaient cinq étages) qui s'imbriquent les unes dans les autres autour d'une église centrale. L'architecture considérée comme un moyen de réforme sociale acquiert ainsi une fonction de socialisation. Dans ce cas précis, elle est la transcription graphique de la protection et de la surveillance et offre un des meilleurs exemples architecturaux de l'idéologie néo-féodale.

34 Lettres écrites des rives de l'Ohio, p. 16.

35 Lezay-Marnésia avait pu, lorsqu'il était membre de la loge des Amis Réunis, faire des recherches sur les petites communautés qui pouvaient offrir un terrain d'entente avec les principes spiritualistes de la franc-maçonnerie. Bethléem répondait en partie à ce critère.

36 Lettres écrites des rives de l'Ohio, pp. 70-72.

37 Ibid, p. 110.

38 On retrouve la même idée chez Chastellux, Voyages, p. 436.

39 Lettres écrites des rives de l'Ohio, p. 36. 